\title{
Relaxation of classical many-body hamiltonians in one dimension
}

\author{
Stefano Lepri \\ Max-Planck-Institut für Physik komplexer Systeme, Nöthnitzer Straße 38, D-01187 Dresden, Germany \\ lepri@mpipks-dresden.mpg.de
}

\begin{abstract}
The relaxation of Fourier modes of hamiltonian chains close to equilibrium is studied in the framework of a simple mode-coupling theory. Explicit estimates of the dependence of relevant time scales on the energy density (or temperature) and on the wavenumber of the initial excitation are given. They are in agreement with previous numerical findings on the approach to equilibrium and turn out to be also useful in the qualitative interpretation of them. The theory is compared with molecular dynamics results in the case of the quartic Fermi-Pasta-Ulam potential.
\end{abstract}

Keywords: Relaxation to equilibrium, Fourier modes, Fermi-Pasta-Ulam model

PACS numbers: 05.70.Ln, 05.45.+b, 63.10.+a

To appear in Phys. Rev. E (1998)

\section{INTRODUCTION AND MOTIVATIONS}

Consider the conceptual experiment where one of the normal modes of an idealized, one-dimensional crystal is excited by means of some external source, in such a way that the system is brought far from thermal equilibrium. After switching off the external perturbation, it will relax again to the equipartition state, described by the canonical measure. The classical question is: how long will it take? Such a basic issue was seriously reconsidered in the light of recent results of contemporary nonlinear dynamics, in particular after the discovery that weakly nonlinear systems may display extremely long relaxation times. The latter are related to an effective "freezing" of some degrees of freedom, i.e. to the slow diffusion in phase space (see for example Ref. [1] for a recent critical discussion and further bibliographical references). Because of their simplicity, hamiltonian chains of oscillators are suitable model systems to discuss the problem, both from the analytical than from the simulation point of view.

Although some theoretical work 2, 2,3] indicates that equipartition in a strict sense is always attained in the thermodynamic limit for chains at finite temperature (or energy per particle), no explicit estimates of the time scales needed are known. The problem has been attached mainly from the numerical side, by looking at the time relaxation of suitable indicators of equipartition among the Fourier modes of the chain [i $[$ []. In particular, recent studies [4] focused on the dependence of relaxation times on the energy density or temperature, and some empirical scaling laws were found. Nonetheless, no quantitative explanation of the latter is sofar given. The need for some analytic clue is even more evident as one consider that the computational limits of simulations can be rapidly reached. This is of course a particularly serious limitation at very low energies, when the interaction among modes and the resulting dissipative effects are extremely weak.

A further motivation comes from the closely related problem of energy transport in such systems. As is well known, the relaxation of fluctuations is strictly connected to transport coefficients, and the existence of slow time scales must be reflected somehow in their properties. Indeed, extensive molecular dynamics studies [9] gave evidence of the divergence of the thermal conductivity associated to the algebraic decay of the correlation function appearing in the corresponding Green-Kubo formula.

The present work aims to give a contribution to the comprehension of both questions by studying the relaxation of chains with an acoustic spectrum. The models we will refer to are introduced Sec. II. When the system is not too far from equilibrium, one can rely on well-established theories, like the perturbative and mode-coupling approximations that are reviewed in Sect. III. Their validity will be compared with the outcomes of numerical simulations for the specific case of a interatomic potential with quartic nonlinearities (Sect. IV). Besides the original motivations, the comparison constitutes a nice test of mode-coupling theory and of its typical features i.e. the existence of long time-tails with nontrivial exponents, and the nonanalytic behaviour of the spectrum of relaxation times. This latter property is actually a peculiarity of nonequilibrium dynamics in one dimension. As it will be hopefully clear, this approach will be extremely useful for the problems at hand, as they provide estimates of the relaxation times of the Fourier modes of the chain. Rather surprisingly, a 
comparison with some previous numerical results will show that the latter are largely the main cause of slow relaxation to equipartition (Sect. V).

\section{ONE-DIMENSIONAL MANY-BODY HAMILTONIANS}

We consider a chain of $N$ anharmonic oscillators and denote with $q_{l}$ the displacement of the $l$-th particle from its equilibrium position. The hamiltonian reads as is

$$
H=\sum_{l=1}^{N}\left[{\frac{p_{l}}{2}}^{2}+V\left(q_{l+1}-q_{l}\right)\right]
$$

where the usual Born-Von Karman boundary conditions $q_{l}=q_{l+N}$ are assumed and the potential energy is of the form

$$
V(x)=\frac{x^{2}}{2}+V_{A}(x)
$$

with $V_{A}$ denoting the anharmonic part of it. We will consider homogeneous lattices, so that all the masses are set equal to unity and $p_{l}=\dot{q}_{l}$. The lattice spacing is also set to unity as well as the harmonic frequency, so that all variables in the following are adimensional. This also means that the sound velocity, as defined in the harmonic approximation, is equal to one.

In the following we will always refer to the energy density $\varepsilon$ (energy per particle) and/or to the corresponding temperature $k_{B} T=1 / \beta$. Obviously, for a strongly nonlinear systems, the two quantities are not in general strictly proportional.

The (complex) amplitudes $Q_{k}$ of the Fourier modes are defined through the usual transformation

$$
Q_{k}=\frac{1}{\sqrt{N}} \sum_{l=1}^{N} q_{l} e^{i \frac{2 \pi k}{N} l}, \quad Q_{-k}=Q_{k}^{*}, \quad k=-\frac{N}{2}+1, \ldots, \frac{N}{2}
$$

Once the hamiltonian is expressed in these new canonical variables, the equations of motion become

$$
\begin{aligned}
& \dot{Q}_{k}=\frac{\partial H}{\partial P_{k}^{*}}=P_{k} \\
& \dot{P}_{k}=-\frac{\partial H}{\partial Q_{k}^{*}}=-\omega_{k}^{2} Q_{k}+\mathcal{F}_{k}
\end{aligned}
$$

with $\mathcal{F}_{k}$ being the interaction force among modes, and we have introduced the usual normal-mode frequencies

$$
\omega_{k}=2\left|\sin \left(\frac{\pi k}{N}\right)\right|
$$

\section{ESTIMATE OF THE RELAXATION TIMES}

The formulation of stochastic equations for the dynamics of the relevant variables is rather customary to describe the relaxation close to equilibrium [10]. The idea is to describe the effective motion of suitable "slow" observables by reducing the level of description. The general strategy involves projection on their subspace, and results in linear non-markovian equations. Whenever a sharp separation of time scales is possible the latter reduce to their markovian limit. The memory term determines the relaxation properties and can be estimated self-consistently. Our aim is to apply the above procedure to the present system. In the following two subsections we summarize the relevant steps. 


\section{A. General setting}

Due to the conservation law of total momentum, we expect that in the present case the slow dynamics should be associated with the long-wavelength Fourier modes $Q_{k}$ with $|k| \ll N / 2$. Moreover, translational invariance implies that each mode is uncorrelated from the others so that we can that consider each mode separately. * Accordingly let us consider the set of $Q_{k}$ and $P_{k}$ as relevant variables and define the projection operator $\mathcal{P}$ acting on the scalar observable $X$ as

$$
\hat{\mathcal{P}} X==\sum_{k}\left[\frac{\left\langle X Q_{k}^{*}\right\rangle}{\left\langle\left|Q_{k}\right|^{2}\right\rangle} Q_{k}+\frac{\left\langle X P_{k}^{*}\right\rangle}{\left\langle\left|P_{k}\right|^{2}\right\rangle} P_{k}\right]
$$

The projection of the equation of motion leads to 10

$$
\begin{aligned}
& \dot{Q}_{k}=P_{k} \\
& \dot{P}_{k}=-\tilde{\omega}_{k}^{2} Q_{k}-\int_{0}^{t} \Gamma_{k}(t-s) P_{k}(s) d s+R_{k}
\end{aligned}
$$

where $R_{k}=(1-\hat{\mathcal{P}}) \dot{P}_{k}$ is the so-called random force which is related to the memory function by the fluctuationdissipation theorem

$$
\Gamma_{k}(t)=\beta\left\langle R_{k}(t) R_{k}^{*}(0)\right\rangle
$$

and the renormalized frequencies are given, for a generic hamiltonian like (1), by

$$
\tilde{\omega}_{k}^{2}=\frac{1}{\beta\left\langle\left|Q_{k}\right|^{2}\right\rangle}=(1+\alpha) \omega_{k}^{2} \quad, \quad \alpha(\beta)=\frac{1}{\beta} \frac{\int e^{-\beta V(x)} d x}{\int x^{2} e^{-\beta V(x)} d x}-1 .
$$

Obviously in the harmonic limit $\alpha \rightarrow 0$, and the usual bare dispersion relation (6) is recovered. Here and in the following we will always deal with bounding potentials so that the integrals in (11) are convergent. The definition (11) amounts then to a renormalization of the sound speed from unity to the temperature dependent value $\tilde{v}=\sqrt{1+\alpha}$.

The main object of study will be the normalized correlation function

$$
\mathcal{G}_{k}(t)=\beta \tilde{\omega}_{k}^{2}\left\langle Q_{k}(t) Q_{k}^{*}(0)\right\rangle
$$

which is defined in such a way that $\mathcal{G}_{k}(0)=1$. It satisfies the equation of motion 10

$$
\ddot{\mathcal{G}}_{k}+\tilde{\omega}_{k}^{2} \mathcal{G}_{k}=-\int_{0}^{t} \Gamma_{k}(t-s) \dot{\mathcal{G}}_{k}(s) d s
$$

Introducing the Laplace transforms $\mathcal{G}_{k}(z)$ and $\Gamma_{k}(z)$ with the definition

$$
\Gamma_{k}(z)=\int_{0}^{\infty} e^{-i z t} \Gamma_{k}(t) d t
$$

one has that (with $\left.\dot{\mathcal{G}}_{k}(0)=0\right)$

$$
\mathcal{G}_{k}(z)=\frac{i z+\Gamma_{k}(z)}{z^{2}-\tilde{\omega}_{k}^{2}-i z \Gamma_{k}(z)}
$$

If the dissipation is small enough with respect to $\tilde{\omega}_{k}$, the transform $\mathcal{G}_{k}(z)$ has two poles close to the real axis in the complex plane approximatively given by

$$
\pm \tilde{\omega}_{k}-\frac{i}{2} \lim _{z \rightarrow \tilde{\omega}_{k}+i 0^{+}} \Gamma_{k}(z),
$$

(at fixed wavenumber and provided that the limit exists). This corresponds to both a shift of the renormalized frequencies and a small damping $\gamma_{k}$, given by the imaginary part of (16) (i.e. by the real part of $\Gamma_{k}\left(\tilde{\omega}_{k}\right)$ ). Accordingly, the inverse of the latter defines a characteristic relaxation time of each Fourier mode.

\footnotetext{
${ }^{*}$ One can easily convince hymself of this statement by computing for instance the correlation $\left\langle Q_{k}(t) Q_{k^{\prime}}^{*}(0)\right\rangle$ and imposing that $\left\langle q_{l}(t) q_{l^{\prime}}(0)\right\rangle$ depends only on $\left|l-l^{\prime}\right|$.
} 


\section{B. The mode-coupling approximation}

The above results are more or less formal manipulations: we obviously need to compute explicitely the memory kernel and the relaxation rates $\gamma_{k}$ by resorting to some approximations. A first conceptual difficulty of the projection approach is the fact that $R_{k}$ does not evolve with the full Liouvillean operator associated with $H$ [10]. One generally bypass the problem by simply replacing

$$
\left\langle R_{k}(t) R_{k}^{*}(0)\right\rangle \rightarrow\left\langle\mathcal{F}_{k}(t) \mathcal{F}_{k}^{*}(0)\right\rangle
$$

where the last average is on the full Gibbs measure. In such a way, it is also implicitely assumed that slow terms possibly contained in $\mathcal{F}_{k}$ are negligible in the thermodynamic limit (see below). A second simplification amounts to factorize multiple correlations so that the resulting approximate expression of the memory kernel $\Gamma_{k}(z)$, together with Eq. (15), constitute a closed system of equations for $\mathcal{G}_{k}$. The latter has to be solved self-consistently.

Let us focus on the Fermi-Pasta-Ulam (FPU) potential

$$
V_{A}(x)=\frac{1}{3} g_{3} x^{3}+\frac{1}{4} g_{4} x^{4}
$$

so that $\mathcal{F}_{k}=\mathcal{F}_{k}^{(3)}+\mathcal{F}_{k}^{(4)}$ with

$$
\begin{aligned}
& \mathcal{F}_{k}^{(3)}=-g_{3} \omega_{k} \frac{1}{\sqrt{N}} \sum_{k_{1}+k_{2}=k} \omega_{k_{1}} \omega_{k_{2}} Q_{k_{1}} Q_{k_{2}} \\
& \mathcal{F}_{k}^{(4)}=-g_{4} \omega_{k} \frac{1}{N} \sum_{k_{1}+k_{2}+k_{3}=k} \omega_{k_{1}} \omega_{k_{2}} \omega_{k_{3}} Q_{k_{1}} Q_{k_{2}} Q_{k_{3}}
\end{aligned}
$$

and where the condition on the indexes of the sum is intended to be modulo $N$ (quasi-momentum conservation). Obviously, this represent a reasonable approximation of a generic anharmonic potential in the limit of small anharmonicity. In the approximation where multiple correlations factorize in products, the memory kernel will be a sum of two terms (averages with an odd number of $Q$ vanish):

$$
\Gamma_{k}(t)=\beta\left[\left\langle\mathcal{F}_{k}^{(3)}(t) \mathcal{F}_{k}^{(3) *}(0)\right\rangle+\left\langle\mathcal{F}_{k}^{(4)}(t) \mathcal{F}_{k}^{(4) *}(0)\right\rangle\right] .
$$

and one can readily evaluate the two contribution from the cubic and quartic terms respectively

$$
\begin{aligned}
\beta\left\langle\mathcal{F}_{k}^{(3)}(t) \mathcal{F}_{k}^{(3) *}(0)\right\rangle & \approx C_{3} \omega_{k}^{2} \frac{1}{N} \sum_{k_{1}+k_{2}=k} \mathcal{G}_{k_{1}}(t) \mathcal{G}_{k_{2}}(t) \\
\beta\left\langle\mathcal{F}_{k}^{(4)}(t) \mathcal{F}_{k}^{(4) *}(0)\right\rangle & \approx C_{4} \omega_{k}^{2} \frac{1}{N^{2}} \sum_{k_{1}+k_{2}+k_{3}=k} \mathcal{G}_{k_{1}}(t) \mathcal{G}_{k_{2}}(t) \mathcal{G}_{k_{3}}(t)
\end{aligned}
$$

where we have defined the two constants

$$
C_{3}=3 \frac{g_{3}^{2}}{\beta(1+\alpha)^{2}} \quad, \quad C_{4}=15 \frac{g_{4}^{2}}{\beta^{2}(1+\alpha)^{3}}
$$

The numerical factors come from the counting all the possible factorizations. Before going further, notice that in the present example we can easily understand the assumption of neglecting slow components in (17). The force $\mathcal{F}_{k}^{(4)}$ contains indeed a term proportional to $Q_{k}\left|Q_{k}\right|^{2} / N$, which is clearly as slow as $Q_{k}$ itself. As assumed, its weight vanishes like $1 / N$, but for any finite $N$, it may be then regarded as one of the sources of finite-size effects in the numerical simulations.

Even with all the above simplifications the theory remains too complicated to be solved. A further assumption is then generally required, namely that the sums in Eqs. (22) and (23) can be replaced with their values at $k=0$ [11. For the cubic case this amounts to set $k_{1}=-k_{2}=k^{\prime}$ in Eq. (22), yielding

$$
\beta\left\langle\mathcal{F}_{k}^{(3)}(t) \mathcal{F}_{k}^{(3) *}(0)\right\rangle \approx C_{3} \omega_{k}^{2} \frac{1}{N} \sum_{k^{\prime}} \mathcal{G}_{k^{\prime}}^{2}(t)
$$


For the quartic term we can also extend the summation only to the small $k$ terms that are almost-resonating, e.g. those for which $k_{1}-k_{2}-k_{3} \approx 0$. This simplification is justified as in the long-time limit only the slowly oscillating contributions should be significative. One can convice hymself that this kind of approximation leads to the same result (25) also for the quartic term, with $C_{3}$ replaced by $C_{4}$. Finally, in the limit $N \rightarrow \infty$, we let $2 \pi k / N \rightarrow q$ and replace the sums with integrals. The above hypotesis that we can set $k=0$ in Eqs. (22) and (23) amounts therefore to say that the memory kernel can be written in the form $\Gamma(q, z)=\nu(z) q^{2}$ for $q \rightarrow 0$ [11]. As a result, one gets the self-consistency relation for $\nu$ from the Laplace transform of Eq. (10)

$$
\nu(z) \approx C \int_{0}^{\infty} d t e^{-i z t} \int \frac{d q}{2 \pi} \mathcal{G}^{2}(q, t)
$$

where $C=C_{3}+C_{4}$. This is readily solved by dimensional arguments [1], and yields

$$
\nu(z) \propto \frac{C}{\sqrt{\nu(z) z}} .
$$

This last relation implies that $\Gamma(q, z) \sim z^{-1 / 3} q^{2}$, so that the limit in Eq. (16) leads to a non-analytic dependence of the relaxation rates for small wavenumber:

$$
\gamma(q) \propto\left(\frac{C^{2}}{\tilde{v}}\right)^{1 / 3} q^{5 / 3} .
$$

Generally speaking, the behaviour of the relaxation rates with the temperature will depend on the specific form of the anharmonic potential. Nevertheless, the $q$-dependence should be the same for all one-dimensional models where the theory applies.

\section{Kinetic vs. hydrodynamic relaxation}

The above self-consistent result is expected to hold for strong anharmonicity and on very long ("hydrodynamic") time scales. On the other hand, in the usual perturbative limit, the system is basically a set of weakly interacting harmonic oscillators with renormalized frequencies. We can then use the perturbation theory in our simple mode-coupling scheme. This basically amounts to neglect the dissipation on the r.h.s. of Eq. (26) and approximate $\mathcal{G}(q, t) \approx \cos \tilde{\omega}(q) t$. In this limit the factorization of correlations becomes exact. Accordingly, Eq. (26) reduces then to a simplified version of the usual perturbative formula [13], where only mode-mode contributions are taken into account. It then follows that the spectrum of the dissipation rates is proportional to $\omega_{k}^{2}$ times the sum of two terms whose magnitude is given by the constants $C_{3}$ and $C_{4}$ respectively. In the limit of low temperatures and/or weak couplings the latter scale as

$$
\begin{aligned}
\frac{\left(g_{3} q\right)^{2}}{\beta(1+\alpha)^{5 / 2}} \approx\left(g_{3} q\right)^{2} \varepsilon & & (\text { from cubic term }) \\
\frac{\left(g_{4} q\right)^{2}}{\beta^{2}(1+\alpha)^{7 / 2}} \approx\left(g_{4} q\right)^{2} \varepsilon^{2} & & \text { (from quartic term) }
\end{aligned}
$$

for small wavenumbers. It is of basic importance to compare this latter ("kinetic") time scale with the one determined in the previous subsection. For example, in the case of the quartic FPU model we have from Eqs. (28) and (29)

$$
\frac{\tau_{\text {hydro }}}{\tau_{\text {kin }}} \sim q^{1 / 3}\left(g_{4} \varepsilon\right)^{2 / 3} \ll 1
$$

This implies that, for small enough $g_{4} \varepsilon$, the initial relaxation stage is dominated by the kinetic time scale, up to some (possibly large) crossover time where the self-consistency effects become relevant. In Section V we will come back on this issue to show its importance for the approach to equilibrium.

\section{MOLECULAR DYNAMICS RESULTS}

As many assumptions are required in the theory, it is important to compare it with the outcomes of numerical simulations. To this aim, we considered the quartic FPU case $\left(g_{3}=0\right)$. In this case, the only relevant parameter is $g_{4} \varepsilon$ and $g_{4} \varepsilon \ll 1$ correspond to the weakly chaotic regime $\llbracket 4$. 
The numerical simulations were performed at constant energy by integrating the equations of motion with a third order symplectic algorithm [14. We generally consider the case where the second constant of motion $P_{0}=\dot{Q}_{0}$ is identically zero (no uniform rotations of the chain). Equilibrium initial conditions were chosen either by assigning random velocities from a Gaussian distribution at the corresponding temperature, or starting from equal mode amplitudes with uniformely distributed random phases. The system is then evolved for a certain transient time in order to start the measurements from a more generic phase-space point as possible. In computing spectra and correlation functions a Fast Fourier Transform routine has been used, and the data are usually averaged over an ensemble of several trajectories (typically between 20 and 200) to reduce statistical fluctuations.

Let us first of all comment on the dynamics of Fourier modes. The correlation of the fluctuating force decays on a characteristic time that is expected to be much shorter than the typical period of long-wavelength modes. Thus, if we neglect memory effects and assume that a single relaxation time dominates, Eq. (9) reduces to its Markovian limit (we come back to the discrete case):

$$
\ddot{Q}_{k}+\gamma_{k} \dot{Q}_{k}+\tilde{\omega}_{k}^{2} Q_{k}=R_{k}
$$

where now the random force is well approximated by a Gaussian white process

$$
\left\langle R_{k}(t) R_{k}^{*}\left(t^{\prime}\right)\right\rangle=\frac{\gamma_{k}}{\beta} \delta\left(t-t^{\prime}\right)
$$

For simplicity, in Eq. (31) we have also neglected the small frequency shift. Eq. (31) explains qualitatively the numerical results of Ref. [12 where the dynamics of the modes was studied for the quartic FPU case. In particular, the slow diffusion of energy observed there is immediately understood as a consequence of the fact that $\gamma_{k} / \tilde{\omega}_{k} \ll 1$ for small $k$. The renormalization of the frequencies, that was proposed on purely phenomenological basis is, in the present context, a straightforward consequence of the projection approach. Furthermore, the effective sound velocity can now be explicitely computed by the definition (11) (at least up to correction from Eq. $(16))$.

At high energy $\left(g_{4} \varepsilon \gg 1\right)$, where relaxation occours on faster time scales, it is relatively easier to perform direct tests of the goodness of Eq. (31). We first verified that the distributions of the real and imaginary parts of $Q_{k}$ and $P_{k}$ are Gaussian. A typical correlation function $\mathcal{G}_{k}$ is reported in Fig. 1. Moreover, we checked that the distribution of amplitudes and phase jumps agrees with what predicted from the approximation Eq.(31) [15]. For completeness, we also measured the dependence of the effective oscillation frequency, and compared it with the expected value given by the definition (11) (see Fig. 2).

Clearly, the crucial point to be checked is the energy and wavenumber dependence of the $\gamma_{k}$. This was accomplished by measuring the initial decay of the envelope of $\mathcal{G}_{k}$ (see Fig. 1), for several $\varepsilon \mathrm{s}$ and $k \mathrm{~s}$. A very good agreement with the mode-coupling prediction is obtained for the dependence of $\gamma_{k}$ on the wavenumber. The data reported in Fig. 3 give a power law with an exponent 1.64, remarkably close to the expected value $5 / 3$. Furthermore, Fig. 4 shows that also the scaling with energy is reasonably obeyed, at least within the limit of our simulations. Obviously, the computations become more and more time-consuming with decreasing temperature due to the rapid increase of relaxation times and finite-size effects (see below). This imposes severe constraints on the accessible lattice lengths and times.

A further analysis has been performed on the quantity

$$
\mathcal{E}=\sum_{k} E_{k} \quad ; \quad E_{k}=\frac{1}{2}\left|P_{k}\right|^{2}+\frac{1}{2} \tilde{\omega}_{k}^{2}\left|Q_{k}\right|^{2}
$$

which is related to the typical indicators used in equipartition studies. For $|k| \ll N / 2$ (small noise amplitudes), we expect from (31) that the effective mode energy $E_{k}$ is ruled by the linear equation [15]

$$
\dot{E}_{k}+\gamma_{k}\left(E_{k}-\left\langle E_{k}\right\rangle\right)=R_{k}^{\prime}
$$

(see the inset of Fig. 作. This means that the asymptotic behaviour of fluctuations $\delta \mathcal{E}$ of the above defined quantity are given (in the thermodynamic limit) by

$$
\langle\delta \mathcal{E}(t) \delta \mathcal{E}(0)\rangle \propto \frac{1}{N} \sum_{k} e^{-\gamma_{k} t} \approx \int \frac{d q}{2 \pi} e^{-\gamma(q) t} \propto \begin{cases}t^{-1 / 2} & \text { for } t \ll t_{C} \\ t^{-3 / 5} & \text { for } t \gg t_{C}\end{cases}
$$


Here $t_{C}$ is the characteristic time scale at which a crossover between the two relaxation behaviours occours. Although its magnitude remains undetermined, we expect it to be very large for small temperature. The crossover should become actually observable in the intermediate-energy region.

The numerical results are in substantial agreement with those predictions. The simulations at $g_{4} \varepsilon=0.05$ (see Fig. 5) indicate a power-law divergence in the spectrum of $\delta \mathcal{E}$ with an exponent -0.5 in the observed domain. The statistical accuracy and the length of the simulation are not sufficient to establish whether the apparent saturation for $\omega<10^{-3}$ is the beginning of the crossover or simply a finite-size effect. A second series of simulations in the intermediate energy range i.e. for $g_{4} \varepsilon=0.45$ is reported in Fig. 6. Despite the strong finite-size effects at low frequency, the spectra seem to approach a power-law behaviour with the expected mode-coupling exponent -0.4 and the data are compatible with (3.5) with a value of $t_{C}$ of the order of $10^{2}$. Since a further support to the validity of the mode-coupling results is also reported in [16], we reasonably conclude that no significative deviations from the theory itself are observed in those type of measurements.

\section{THE PROBLEM OF RELAXATION TO EQUIPARTITION}

At this point we want now to discuss some consequences of the above analysis on the so called FPU-problem. Let us first focus again on the case of a purely quartic nonlinearity that has been intensively (re)studied by several authors in recent years [4,6,8]. The numerical experiments have been performed by feeding the initial energy in a packet of modes and looking at the decay in time of suitable indicators of equipartition (see the quoted references for details).

In the case when the initial excitation is around a long-wavelength mode of wavenumber $q_{*}$, and the system is not too far from equipartition, we expect, from the discussion of Section III, that the slowest time scale will be of the order of

$$
\tau_{E}=\left\{\begin{array}{ll}
\left(g_{4} \varepsilon q_{*}\right)^{-2} & \text { for } g_{4} \varepsilon \ll 1 \\
\left(g_{4} \varepsilon\right)^{-1 / 4} q_{*}^{-5 / 3} & \text { for } g_{4} \varepsilon \gg 1
\end{array} .\right.
$$

The subscript $E$ is precisely meant to remark that it refers to the fluctuations close to the equilibrium state. The behaviour in the high-energy limit is obtained by means of Eq. (28) taking into account that $\varepsilon$ is roughly proportional to the temperature, as well as the fact that $(1+\alpha)$ grows with the square root of the temperature itself (see Eq. (11)).

Indeed, the predicted regimes have been numerically observed 沺. A convincing numerical evidence of scaling laws (36) has been recently reported in Ref. [8]. This seem to suggest that the linear theory already captures the quantitative features. Moreover, qualitatively similar results were found for different potentials in Ref. [17].

For what concerns the case of larger $q_{*}$, the simulations reported in Ref. [4] also showed that relaxation can be one or two orders of magnitude faster than in the previous situation. Even if the theory presented here applies better to the case of small $q_{*}$, it is worth to remark that its consequences are also consistent with this observation.

Obviously, the very initial stage of the approach to equilibrium may well occour on a different time scale. In the present framework, the latter should be interpreted as a real "partial equilibration" time scale $\tau_{N E}$. On the other hand, it seem rather reasonable that $\tau_{N E}$ will be strongly dependent on the chosen class of initial conditions and on the specific form of the potential. As a matter of fact, $\tau_{N E}$ is determined by a pure nonequilibrium dynamics and is of course unaccessible to the linear theory presented here. Despite of this, the present results are useful to identify this initial stage. As an example, we are now able to make an instructive comparison with the numerical estimates of $\tau_{N E}$. In Ref. [6] it is in fact found that, for long-wavelength excitations $\left(q_{*} \ll 1\right)$, $\tau_{N E} \sim N^{1 / 2}\left(q_{*} \varepsilon\right)^{-1}$, so that

$$
\frac{\tau_{N E}}{\tau_{E}} \sim \varepsilon N^{1 / 2} q_{*} \ll 1
$$

for small $q_{*}$ and/or small energy. We can then conclude that the equilibration process is, at least for this class of initial conditions, mainly dominated by the linear regime. In other words, we can naturally understand it as an initial fast relaxation to a quasi-equilibrium state followed by the slow diffusion of energy from the long-wavelength modes.

As already mentioned, the mechanisms determining $\tau_{N E}$ may be however rather complex and of very different nature depending on the initial state. An example, is the case of zone-boundary initial conditions $\left(\left|q_{*}\right| \approx \pi\right)$. Those rapidly decay into localized chaotic excitations [19],8], whose lifetime mainly determines the time to reach 
the quasi-thermalized state. Nevertheless, the comparison of our results with the numerics may indicate that such lifetime is, even in this case, considerably shorter than that of relaxation of Fourier modes $[8]$.

The high-energy scaling of Eq. (36) can be generalized to an algebraic potential of the form $V_{A}(x)=g_{n} x^{n}$, with $n$ being an even integer. In this case, by extending Eqs. (24) and (28), it is found that $\tau_{E}$ is proportional to $\varepsilon^{(1 / n-1 / 2)}$.

Finally let us comment on the the FPU model with purely cubic potential $\left(g_{4}=0\right)$. A recent numerical study [18] showed a clear evidence for a divergence as $\varepsilon^{-3}$ of the relaxation time. Nevertheless only chains as short as $N=32$ were considered, and it is not completely clear if the systems is above the equipartition threshold [3]. A more detailed analysis would of course be desirable to check the dependence of this scaling on $N$ and to compare the results with the ones presented here.

\section{CONCLUSIONS}

The simple mode-coupling approach provided a valuable amount of qualitative and quantitative information on the relaxation times of the Fourier modes in an hamiltonian chain as (11). In particular, a clear physical interpretation of them can be achieved. For the paradigmatic example of the quartic FPU model, the crossover between the two scaling regions at $g_{4} \varepsilon \approx 0.2$ (see again Fig. (4), could be regarded as the temperature threshold beyond which self-consistency and "hydrodynamic" effects play a major role. Remarkably, such a scale is roughly equal to the so-called strong stochasticity threshold $\left(g_{4} \varepsilon \approx 0.1\right)$ above which new dynamical effects (i.e. faster diffusion in phase space) are believed to appear [4]. From this point of view it would be challenging to try to connect the present results with strictly dynamical properties.

The explicit estimates given in the present work turned out to explain several previous results on the approach to equilibrium at finite temperature. As esemplified above, they are a precious information for the numerical study. For example, the slow temporal decay of the equipartition indicators may be better understood having realized the existence of the long-time tails like those of Figs. . and 6 . Indeed, it is clear that the slow diffusion of energy has to be taken into account when studying the relaxation from an arbitrary initial condition. More generally, such time scales should be always considered in practice when performing simulations with chains of large sizes.

Although the existence of long-time tails is not surprising for a low-dimensional system, the substantial agreement between the theory and numerical simulation is nonetheless a relevant result by itself. Actually, the direct verification of mode-coupling theories in one dimension is still current object of study, for example in the field of lattice gases [20]. Furthermore, the validity of such theories is not granted in general. It is in fact known that they fail in predicting the characteristic relaxation times of spin-waves of the Heisenberg model in one and two dimensions [21].

Finally, let us remark that the theory allows to estimate the long-time tails of the heat-flux correlation, which is directly related to the thermal conductivity of the chain. This allows to explain quantitatively the divergence of such transport coefficient observed in the numerical simulations [9.16].

\section{ACKNOWLEDGMENTS}

I acknowledge useful discussions with Roberto Livi, Antonio Politi, Stefano Ruffo and Alessandro Torcini. Thanks also to Jochen Rau and Wolfram Just for having clarified to me several issues of the projection method and for the interest demonstrated towards this work and a careful reading of the manuscript.

[1] G. Benettin, Prog. Theor. Phys. Suppl. 116, 207 (1994).

[2] G. Tsaur, J. Wang, Phys. Rev. E 544657 (1996).

[3] D.L. Shepelyansky, Nonlinearity 10, 1331 (1997).

[4] M. Pettini, M. Cerruti-Sola, Phys. Rev. A 44975 (1991).

[5] H. Kantz, R. Livi, S. Ruffo, J. Stat. Phys. 76, 627 (1994).

[6] J. DeLuca, A.J. Lichtenberg, S. Ruffo, Phys. Rev. E 512877 (1995). 
[7] G. Parisi, Europhys. Lett. 40, 357 (1997).

[8] T. Cretegny, T. Dauxois, S. Ruffo, A. Torcini, cond-mat/9709204 to appear in Physica D.

[9] S. Lepri, R. Livi, A. Politi, Phys. Rev. Lett. 78, 1896 (1997).

[10] R.Kubo, M.Toda, N. Hashitsume, Statistical Physics II, Springer Series in Solid State Sciences, Vol. 31 (1991).

[11] Y. Pomeau, R. Résibois, Phys. Rep. 1963 (1975).

[12] C. Alabiso, M.Casartelli, P.Marenzoni, J. Stat. Phys. 79, 451 (1995).

[13] See e.g. T.H.K. Barron, M.L. Klein in Dynamical properties of Solids, G.K. Horton and A.A. Maradudin eds. North Holland (1974).

[14] L. Casetti, Phys. Scr. 51, 29 (1995).

[15] R.L. Stratonovich Topics in the Theory of Random Noise, Gordon and Breach, New York (1963).

[16] S. Lepri, R. Livi, A. Politi, Europhys. Lett. 43, 271 (1998).

[17] K. Yoshimura, Physica D 104, 148 (1997).

[18] L. Casetti, M. Cerruti-Sola, M. Pettini, E.G.D. Cohen, Phys. Rev. E 556566 (1997).

[19] V.M. Burlakov, S. Kiselev, Sov. Phys. JETP 72, 854 (1991).

[20] T. Naitoh, M.H. Ernst, M.A. van der Hoef, D. Frenkel, Phys. Rev. E 47, 4098 (1993).

[21] G. Reiter, Phys. Rev. B 21, 5356 (1980). 


\section{FIGURES}

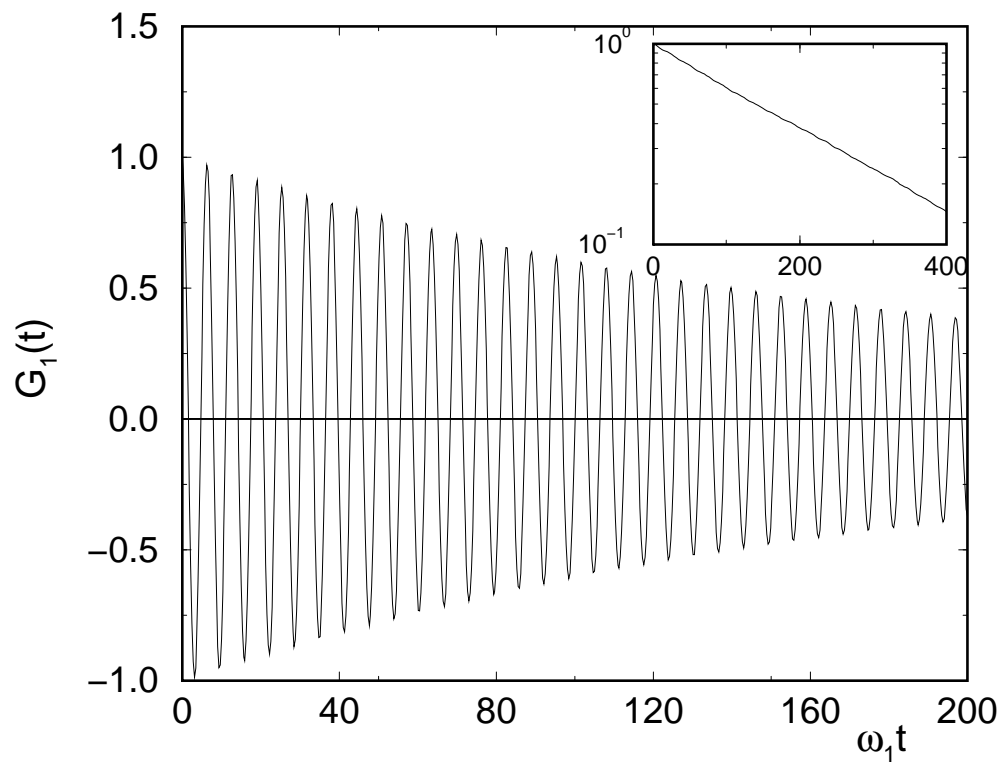

FIG. 1. The normalized autocorrelation $\mathcal{G}_{1}(t)$ for the quartic FPU model with $g_{4} \varepsilon=8.8(T=11.07), N=256$. The inset show the autocorrelation of the fluctuations of the mode energy $E_{1}$. 


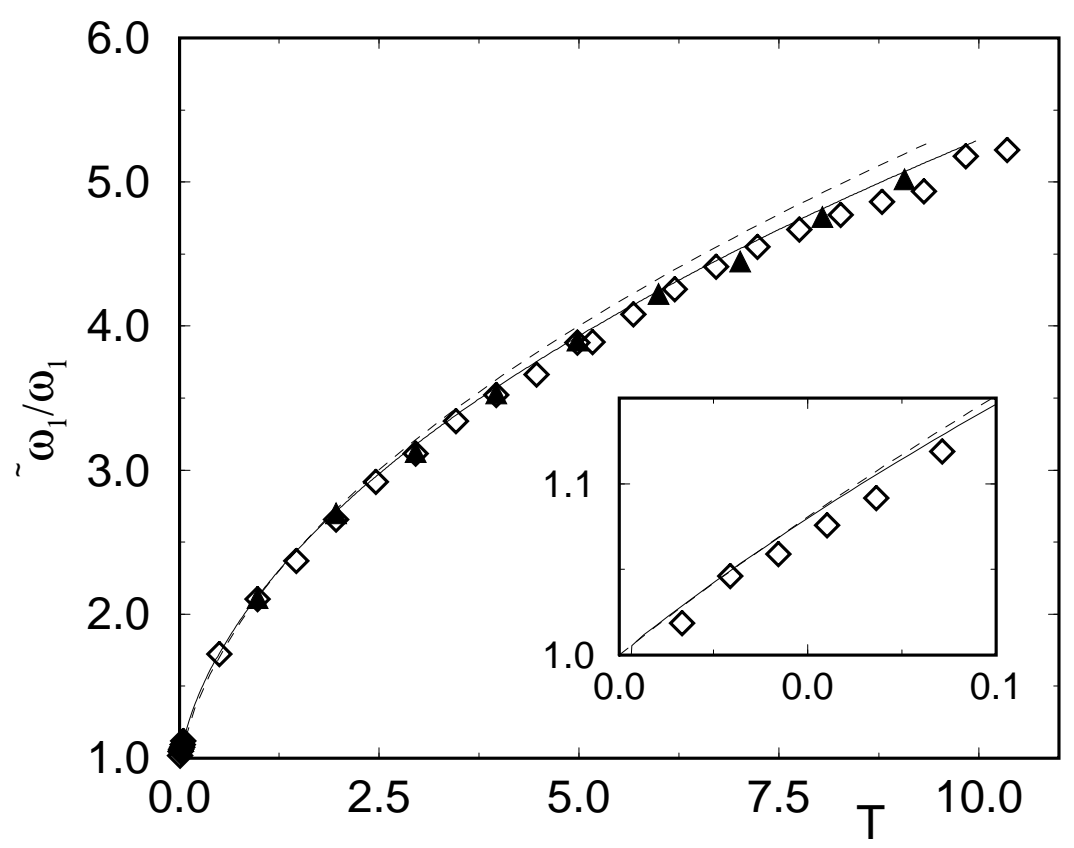

FIG. 2. Comparison between the numerical and expected renormalized frequencies. The symbols are obtained measuring the oscillation period of $\mathcal{G}_{1}$ for $N=64,128$ (diamonds and triangles respectively). The solid line is the theoretical value, Eq. (11) and the dashed one is the empirical formula of Ref. [12]. The small systematic deviations are due to the small frequency shifts expected from (16).

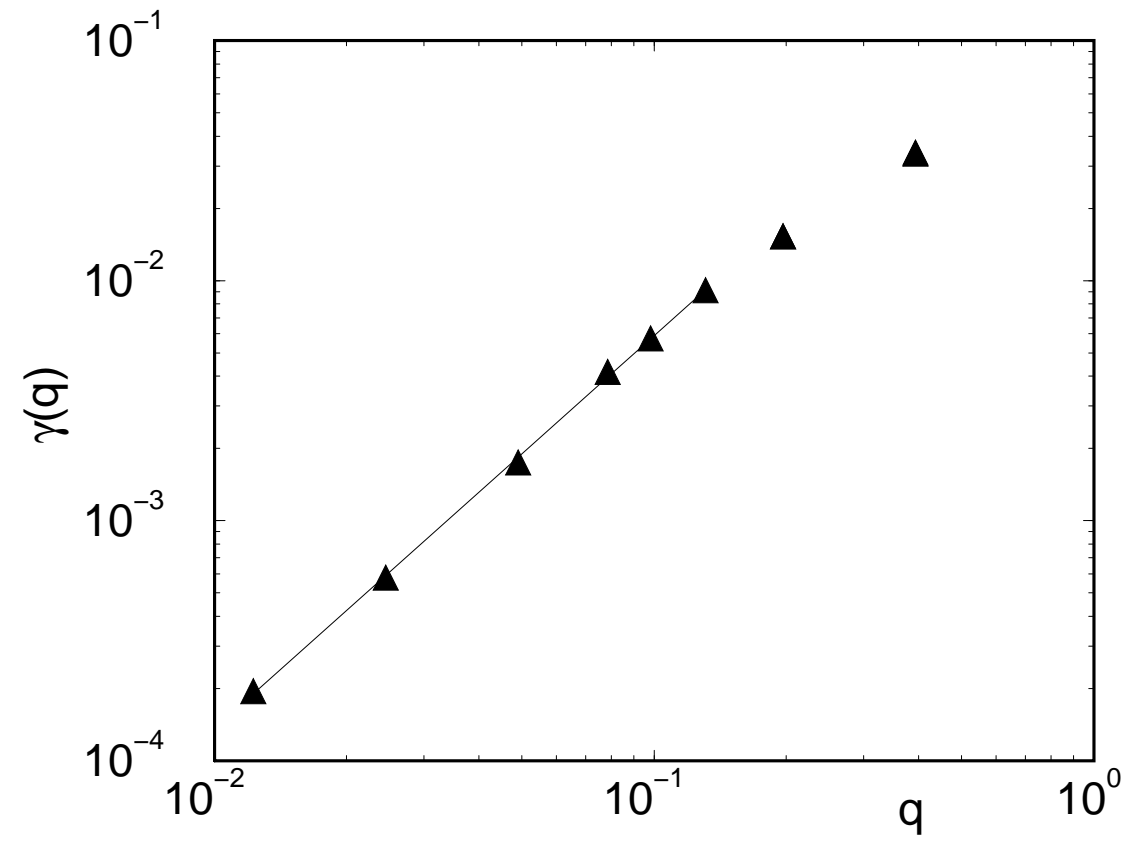

FIG. 3. The wavenumber dependence of the relaxation rates $\gamma(q)$ at $g_{4} \varepsilon=8.8$ for the quartic FPU potential. All the points were obtained from the initial decay of the envelope of $\mathcal{G}_{1}$ for increasing values of $N$ up to $N=2048$. The solid line is a power-law fit $q^{1.64}$. 


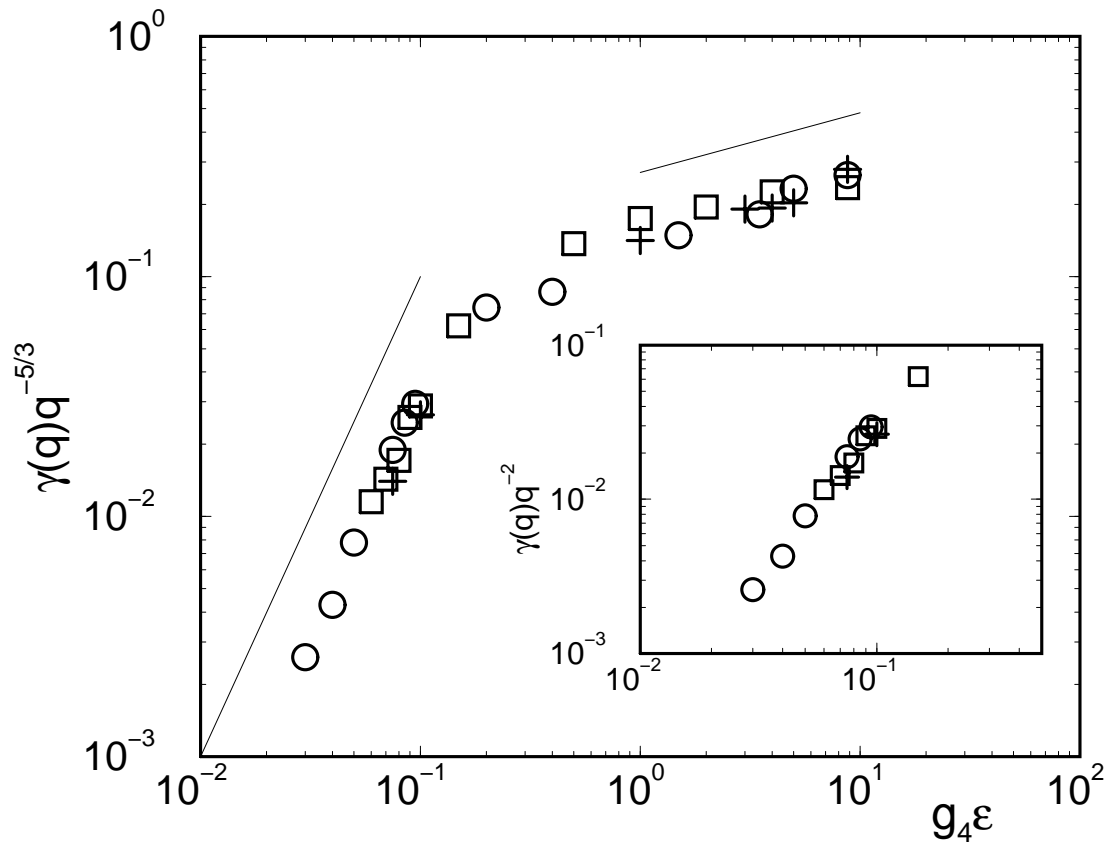

FIG. 4. The scaling of the relaxation rates with the energy density $\varepsilon$ for $N=64,128,256$ (circles, squares and pluses respectively). The inset shows the low energy part. Straight lines correspond to $\varepsilon^{2}$ and $\varepsilon^{1 / 4}$ respectively.

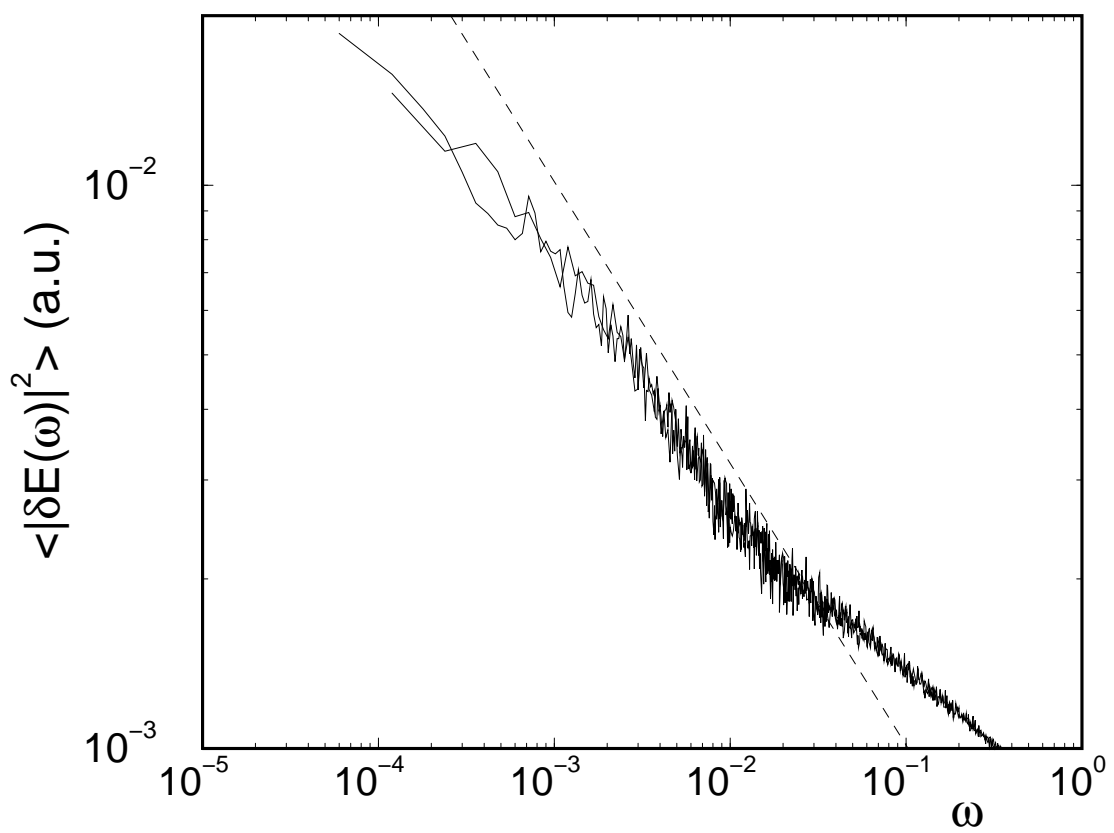

FIG. 5. Low-frequency part of the spectrum of $\delta \mathcal{E}$ for the quartic FPU model for $g_{4} \varepsilon=0.05$. The solid lines refer to simulations with $N=2048,4096$, the dashed line correspond to a $\omega^{-1 / 2}$. A power-law fit gives an exponent $-0.49 \pm 0.01$ for $10^{-3}<\omega<10^{-2}$. 


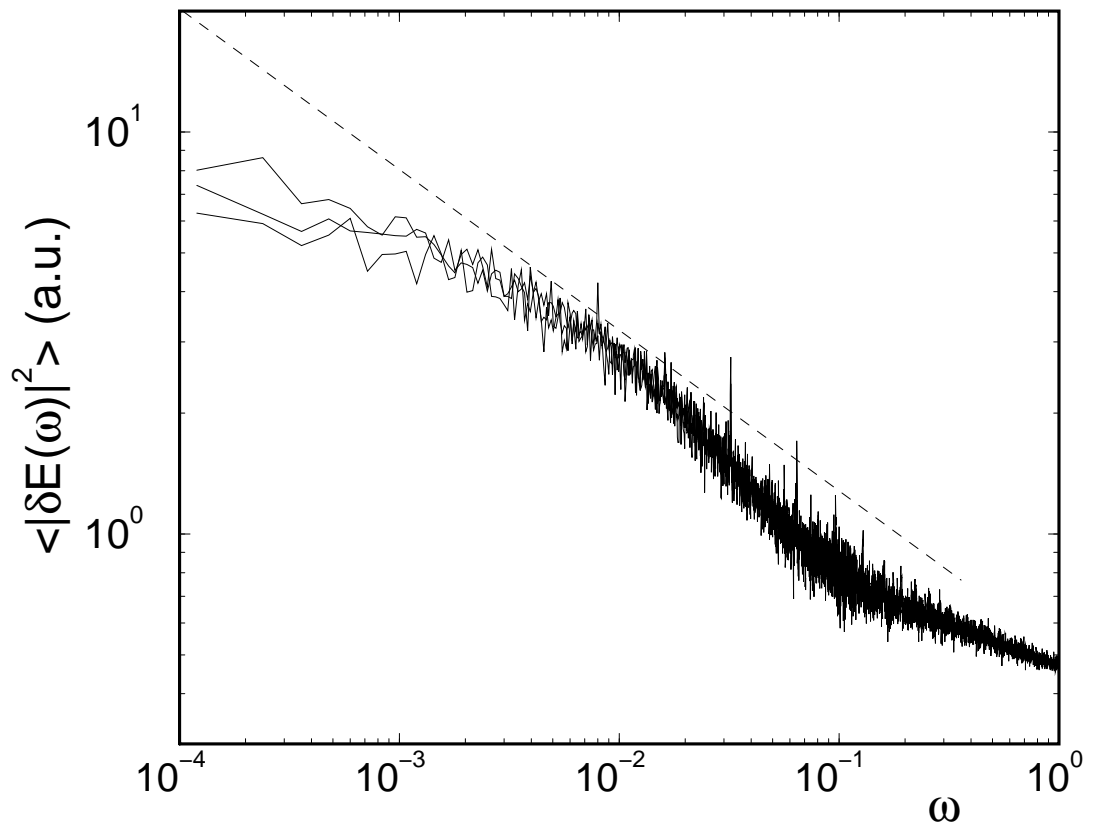

FIG. 6. Same as Fig. 月 but for $g_{4} \varepsilon=0.45$. The curves refer to $N=512,1024,2048$ (from bottom to top), the dashed line corresponds to the expected asymptotic law $\omega^{-2 / 5}$. 